\title{
Charge-order driven multiferroic and magneto-dielectric properties of rare earth manganates
}

\author{
CLAUDY RAYAN SERRAO ${ }^{1,2, *}$, JYOTI RANJAN SAHU ${ }^{1}$ and ANIRBAN GHOSH ${ }^{1,2}$ \\ ${ }^{1}$ Chemistry and Physics of Materials Unit, Jawaharlal Nehru Centre for Advanced Scientific Research, \\ Bangalore 560 064, India \\ ${ }^{2}$ Materials Research Centre, Indian Institute of Science, Bangalore 560 012, India
}

MS received 23 January 2009

\begin{abstract}
Charge-order driven magnetic ferroelectricity is shown to occur in several rare earth manganates of the general formula, $\operatorname{Ln}_{1-x} \mathbf{A}_{x} \mathrm{MnO}_{3}(\mathrm{Ln}=$ rare earth, $\mathrm{A}=$ alkaline earth). Charge-ordered manganates exhibit dielectric constant anomalies around the charge-ordering or the antiferromagnetic transition temperature. Magnetic fields have a marked effect on the dielectric properties of these compounds, indicating the presence of coupling between the magnetic and electrical order parameters. Magneto-dielectric properties are retained in small particles of the manganates. The observation of magneto-ferroelectricity in these manganates is in accordance with theoretical predictions.
\end{abstract}

Keywords. Multiferroics; magnetoelectric effect; magnetocapacitance; rare earth manganates.

\section{Introduction}

Materials which exhibit both magnetic and electrical ordering are attracting great interest in the last few years, partly because of their technological potential. Besides a range of possible device applications, the science of these materials is truly fascinating (Prellier et al 2005; Eerenstein et al 2006; Cheong and Mostovoy 2007; Ramesh and Spaldin 2007; Rao and Serrao 2007). Multiferroics are materials in which ferromagnetism, ferroelectricity and ferroelasticity occur in the same phase. This implies that they possess spontaneous magnetization which can be reoriented by an applied magnetic field, a spontaneous polarization which can be reoriented by an applied electric field and a spontaneous deformation which can be reoriented by an applied stress. It is, however, customary to exclude ferroelasticity and only consider magnetic and ferroelectric characteristics. Multiferroics possess neither spatial inversion symmetry nor time-reversal symmetry.

It is generally difficult to find materials that are magnetic as well as ferroelectric, since conventional ferroelectricity occurs when the metal ions have empty $d$-orbitals. Magnetism, on the other hand, occurs in materials containing cations with partially filled $d$-orbitals. Magnetoelectrics are simultaneously ferromagnetic and ferroelectric in the same phase, with coupling between the two orders. Magnetoelectric coupling describes the influence of a magnetic (electric) field on the polarization (magnetiza-

\footnotetext{
*Author for correspondence (claudy@jncasr.ac.in)
}

tion) of a material. Magnetoelectric coupling can exist independent of the nature of the magnetic and electrical order parameters. It is an independent phenomenon which may not necessarily arise in materials that are both magnetically and electrically polarizable. Since coexistence of magnetic and ferroelectric ordering is not favoured, materials with such properties arising from alternative mechanisms are being sought in recent years.

One of these mechanisms is tilting of $\mathrm{MnO}_{5}$ trigonal bypyramids giving rise to ferroelectricity as in the case of $\mathrm{YMnO}_{3}$ (Van Aken et al 2004). Bismuth compounds such as $\mathrm{BiMnO}_{3}$ and $\mathrm{BiFeO}_{3}$ exhibit ferroelectric properties because of the stereochemical activity of the Bi lone pair (Seshadri and Hill 2001), while in $\mathrm{TbMnO}_{3}$, spiral magnetic ordering is the source of ferroelectricity (Kimura et al 2003). One of the new mechanisms is to induce ferroelectricity and magnetoelectric effects in oxide materials is cation charge-ordering as in the case of some of the rare earth ferrites of the type, $\mathrm{LnFe}_{2} \mathrm{O}_{4}(\mathrm{Ln}=$ rare earth) (Serrao et al 2007). In this article we shall discuss multiferroicity and magnetoelectric effects in rare earth manganates induced by charge-ordering as theoretically predicted by Efremov et al (2004).

\subsection{Charge-ordering in rare earth manganates}

Rare earth manganates of the type, $\mathrm{Ln}_{(1-x)} \mathrm{A}_{x} \mathrm{MnO}_{3}(\mathrm{Ln}=$ rare earth ion, and $\mathrm{A}=$ alkaline earth ion), which crystallize in the perovskite structure were investigated several years ago by Wollan and Koehler (1955). However, it was the discovery of colossal magneto resistance (CMR) that has 
attracted recent attention to these materials (Rao and Raveau 1998; Salamon and Jaime 2001; Tokura 2006). In the parent $\mathrm{LaMnO}_{3}$, the $\mathrm{Mn}$ ions exist in a 3+ state, while $\mathrm{La}_{(1-x)} \mathrm{Ca}_{x} \mathrm{MnO}_{3}$ contains both $\mathrm{Mn}^{3+}$ and $\mathrm{Mn}^{4+}$ ions. The phase diagram of this system reveals a large number of phases with different electronic properties. $\mathrm{LaMnO}_{3}$ exhibits a spin order of an A-type antiferromagnet. This is accompanied by the orbital order which is coupled to the lattice distortion due to the Jahn-Teller (JT) effect. The JT distortion looses long-range order around $750 \mathrm{~K}$. At low temperatures, the combination of orbital ordering and JT distortion leads to a ferromagnetic (FM) exchange interaction between the spins in the $a b$ plane, and a antiferromagnetic (AFM) interaction between the planes, resulting in an $A$-type AFM arrangement.

Doping the rare earth manganates with alkaline earth ions such as $\mathrm{Ca}^{2+}$ and $\mathrm{Sr}^{2+}$ gives rise to charge ordering wherein the +3 and +4 charges of the $\mathrm{Mn}$ ions are arranged in a periodic fashion (figure 1) (Rao 2000). The chargeordered state occurs commonly in the manganates containing small $\mathrm{Ln}$ and $\mathrm{A}$ ions, and disappears at high temperatures. For example, in $\mathrm{Pr}_{0.6} \mathrm{Ca}_{0.4} \mathrm{MnO}_{3}$, charge ordering occurs below $230 \mathrm{~K}\left(T_{\mathrm{CO}}\right)$. It shows AFM order below $170 \mathrm{~K}$, where both antiferromagnetism and charge order coexist (figure 2) (Lees et al 1995; Tomioka et al 1996). At low temperatures, this manganate is an insulator. Application of magnetic fields destroys the charge-ordered state. In contrast, $\mathrm{La}_{0.7} \mathrm{Ca}_{0.3} \mathrm{MnO}_{3}$ does not show charge ordering, although it enters a FM metallic phase below a Curie temperature of $230 \mathrm{~K}$, showing a paramagnetic (PM) insulating behaviour above this temperature. This is a typical manganate composition exhibiting colossal magnetoresistance (Rao and Raveau 1998). In the Srbased $\mathrm{La}_{0.7} \mathrm{Sr}_{0.3} \mathrm{MnO}_{3}$, the high temperature PM phase is metallic. Note that the average radius of the $A$-site cations, $\left\langle r_{A}\right\rangle$, is larger in these two lanthanum manganates

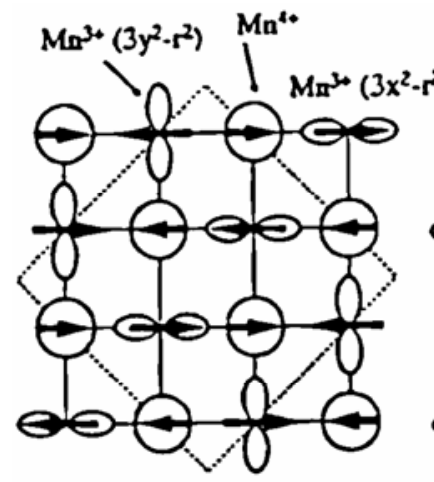

(a)

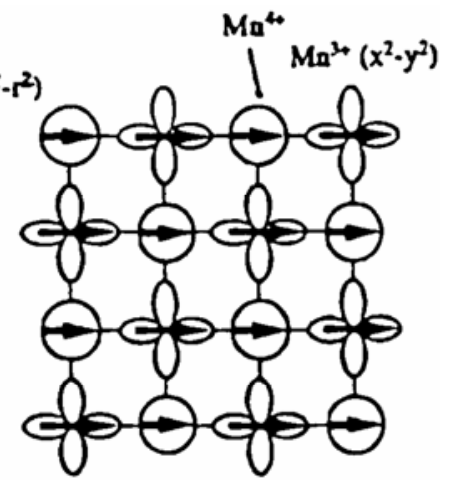

(b)
Figure 1. Ordering of charge, spin and orbital in doped manganates: (a) CE-type order, the dashed line shows the unit cell and (b) $A$-type order. In both figures, circles correspond to $\mathrm{Mn}^{4+}$ (Rao 2000). than in $\mathrm{Pr}_{0.7} \mathrm{Ca}_{0.3} \mathrm{MnO}_{3}$. An important aspect of the doped manganates is that, when the size of the $A$-site cation (or the $e_{\mathrm{g}}$ bandwidth) is large enough, electrons of the $\mathrm{Mn}^{3+}$ hop to $\mathrm{Mn}^{4+}$ by the double-exchange (DE) mechanism (Rao and Raveau 1998; Salamon and Jaime 2001; Tokura 2006). The DE mechanism plays a crucial role in the occurrence of metallicity and ferromagnetism in these materials. The phenomenon of CMR is explained by invoking the DE mechanism along with certain competing interactions involving phonons. Charge ordering has an effect opposite to that of DE, since it localizes electrons creating an AFM insulating state.
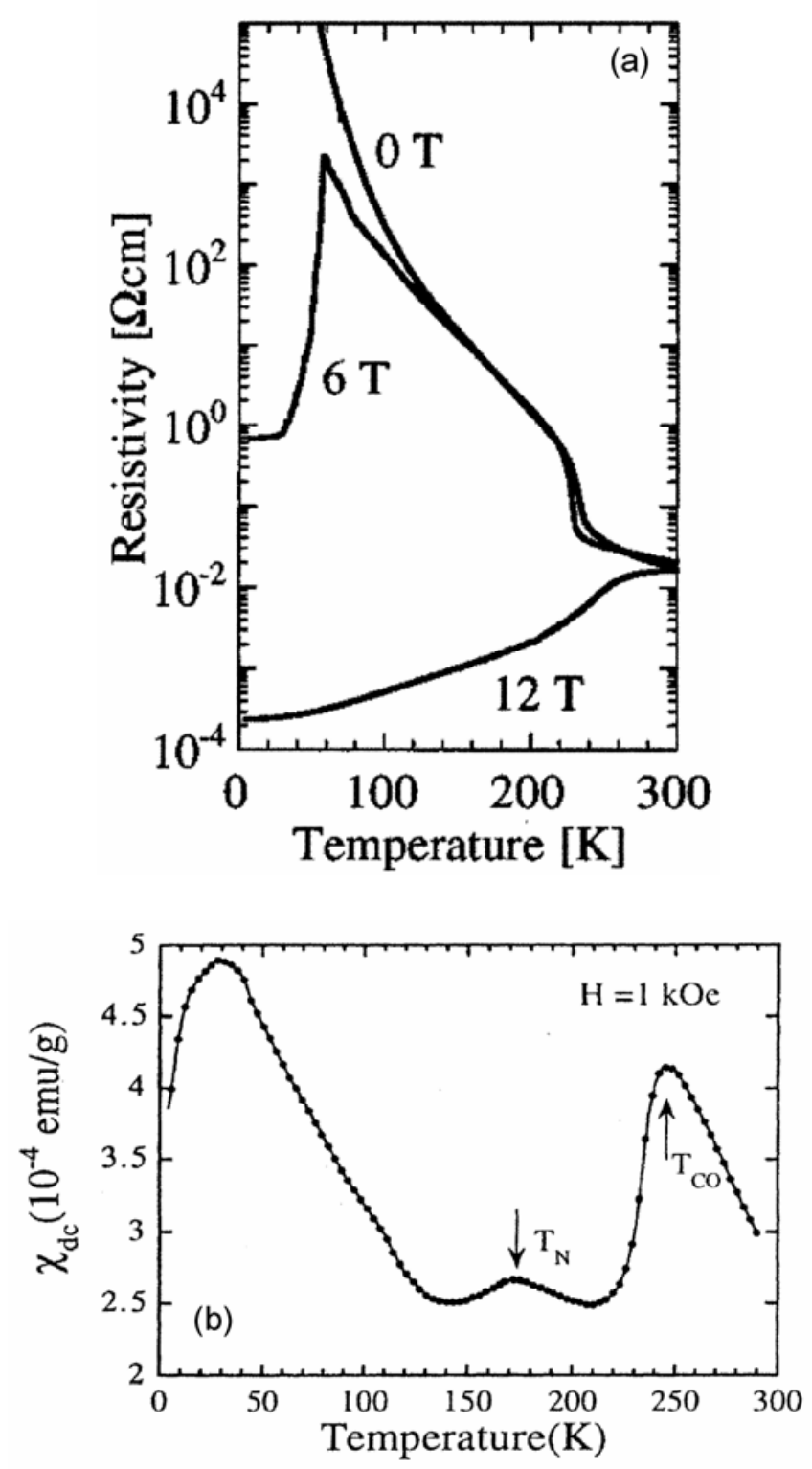

Figure 2. Temperature variation of (a) resistivity and (b) magnetic susceptibility of $\operatorname{Pr}_{0.6} \mathrm{Ca}_{0.4} \mathrm{MnO}_{3}$. The increase in magnetization at low temperatures $(<100 \mathrm{~K})$ is due to electronic phase separation (Lees et al 1995; Tomioka et al 1996). 
Half-doped rare earth manganates show many interesting types of electronic order. For example, $\mathrm{Nd}_{0.5} \mathrm{Sr}_{0.5} \mathrm{MnO}_{3}$, which is a FM metal around room temperature undergoes a transition to a charge-ordered AFM insulator state around $150 \mathrm{~K}$ (figure 3) (Kuwahara et al 1995). At low temperatures, the charge-ordered state is associated with a CE-type AFM order (figure 1), orbital order as well as a pattern due to JT distortions. The magnetic order consists of $a b$ planes stacked along the $c$-axis with an AFM coupling between spins on neighbouring planes and is different from the $A$-type order. Note that there is no charge ordering in an $A$-type antiferromagnet unlike in a CE-type antiferromagnet. Generally, orbital and spin orders which are not accompanied by charge order show $A$-type antiferromagnetism.

The presence of charge order and orbital order is readily found experimentally by a study of the structures of the manganates (figure 4) (Vogt et al 1996; Woodward et al 1999). For example, the charge-ordered structure of $\mathrm{Nd}_{0.5} \mathrm{Sr}_{0.5} \mathrm{MnO}_{3}$ consists of distorted oxygen octahedra with zigzag chains with alternate long and short $\mathrm{Mn}-\mathrm{O}$ bonds. In terms of the chemistry, the mean $A$-site cation radius $\left\langle r_{A}\right\rangle$ is expected to play an important role. This arises from the fact that the bandwidth of the $e_{\mathrm{g}}$ electrons of $\mathrm{Mn}$ ions is affected by the size of the $A$-site cation. That is, increasing $\left\langle r_{A}\right\rangle$ is equivalent to increasing the hydrostatic pressure, which increases the $\mathrm{Mn}-\mathrm{O}-\mathrm{Mn}$ bond angle and consequently the bandwidth. Detailed studies of the effect of $\left\langle r_{A}\right\rangle$ on the electronic order have been reported in the literature (Rao 2000). Manganates with very large $\left\langle r_{A}\right\rangle$ tend to be FM and metallic, with the Curie temperature $T_{\mathrm{C}}$ increasing with $\left\langle r_{A}\right\rangle$. Charge ordering is also strongly affected by $\left\langle r_{A}\right\rangle$, with a smaller $\left\langle r_{A}\right\rangle$ stabilizing the charge-ordered state at low temperatures. It is evident that the tuning of $\left\langle r_{A}\right\rangle$ provides an important means of controlling the electronic order of the manganate. Furthermore, charge ordering can be destroyed to obtain a FM metallic state (Rao 2000) by applying magnetic fields (figures 3 and 5) (Kuwahara et al 1995) or electric fields (figure 6) (Rao et al 2000). Chemical doping (e.g. by ruthenium) can also produce similar effects (Rao 2000). Two types of charge ordering can be distinguished in rare earth manganates. These are site-centred charge ordering ( $\mathrm{SCO})$ and bond-centred charge ordering (BCO) which occur around $x=0.5$ and $x=0.4$ compositions. The two types of charge order are depicted in figure 7 (Efremov et al 2004).

\subsection{Electronic phase separation in rare earth manganates}

There is considerable experimental evidence to indicate that several correlated oxides are electronically inhomogeneous (figure 8). This is manifested best in the rare earth manganates (Dagotto 2005; Shenoy et al 2006;
Shenoy and Rao 2008). Materials with chemical inhomogeneity consist of different spatial regions with different electronic orders. This phenomenon has come to be known as electronic phase separation. The different regions can be static or dynamic and can be tuned by the application of external stimuli like a magnetic field or an electrical field. Moreover, the size scale of these inhomogeneities can vary from nanometers to as large as micrometers.

Investigations by several experimental probes show the presence of electronic inhomogeneities. When the lengthscale of the inhomogeneities is large (a few hundred nanometers), structural and magnetic information from the X-ray and neutron diffraction patterns show distinct signatures. Local probes such as NMR and Mössbauer spectroscopies are used to probe inhomogeneities at a smaller length-scale. Magnetization and transport measurements also indicate the presence of inhomogeneities.

Wollan and Koehler (1955) showed many years ago the presence of electronic inhomogeneities, by the observation of both FM and AFM peaks in the magnetic structure of $\mathrm{La}_{1-x} \mathrm{Ca}_{x} \mathrm{MnO}_{3}$ by neutron scattering. Neutron diffraction studies of Woodward et al (1998) on $\mathrm{Nd}_{0.5} \mathrm{Sr}_{0.5} \mathrm{MnO}_{3}$ shows that this material first becomes $F M$ at $250 \mathrm{~K}$, partially transforms to an $A$-type AFM phase around $220 \mathrm{~K}$, followed by a transformation of a substantial fraction to a CE-type AFM phase at $150 \mathrm{~K}$. The CE-type
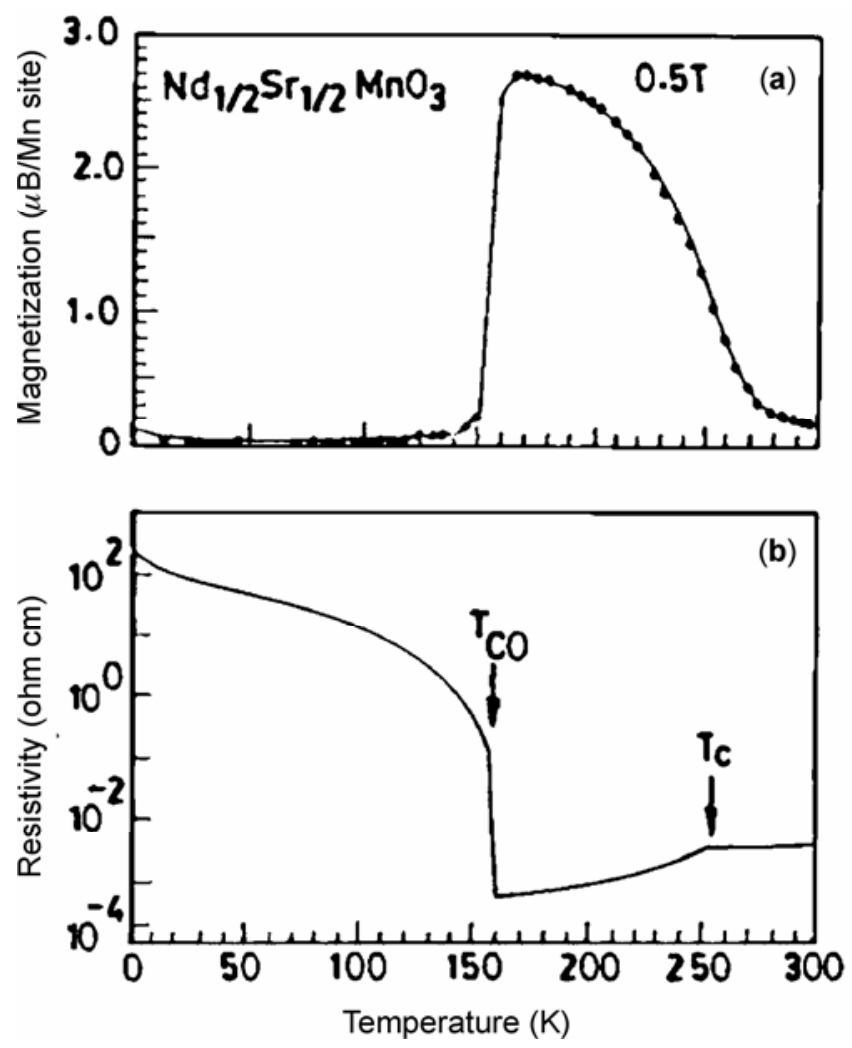

Figure 3. Temperature variation of (a) the magnetization and (b) the resistivity of $\mathrm{Nd}_{0.5} \mathrm{Sr}_{0.5} \mathrm{MnO}_{3}$ (Kuwahara et al 1995). 
(a)

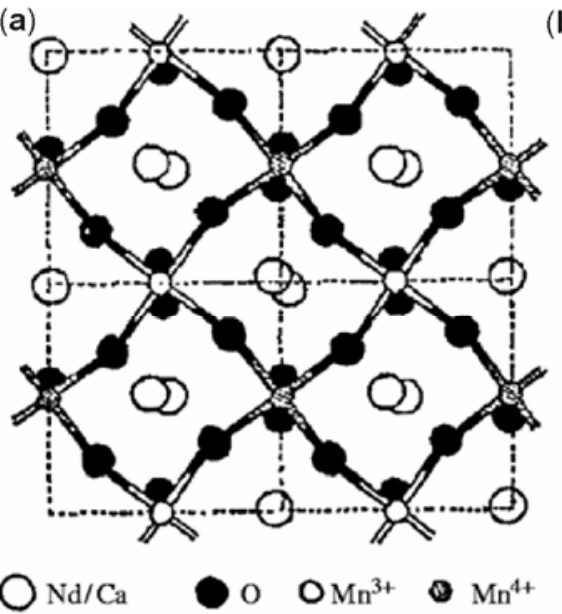

(b)

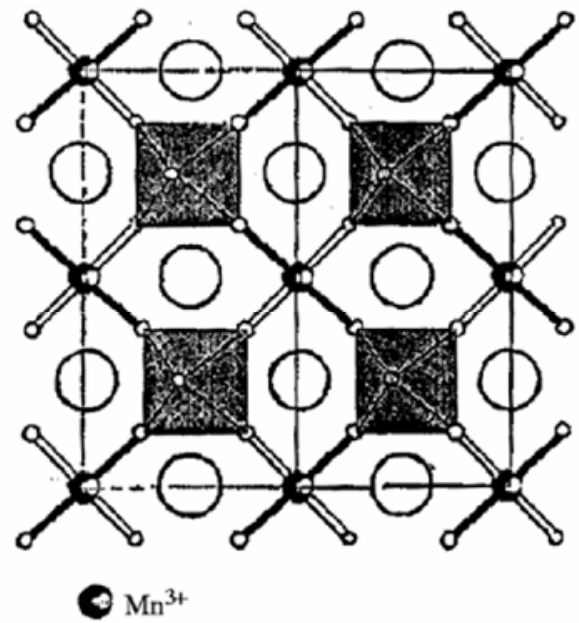

Figure 4. (a) Structure of charge-ordered $\mathrm{Nd}_{0.5} \mathrm{Sr}_{0.5} \mathrm{MnO}_{3}$ in the $a b$ plane at $10 \mathrm{~K} . \mathrm{Mn}^{4+}$ ions are located at $(1 / 2,0,0)$ and $\mathrm{Mn}^{3+}$ are located at $(0,1 / 2,0)$. The structure contains zigzag chains with alternate long and short $\mathrm{Mn}-\mathrm{O}$ bonds. (b) The same structure as given in (a), shown in polyhedral representation (Vogt et al 1996; Woodward et al 1999).

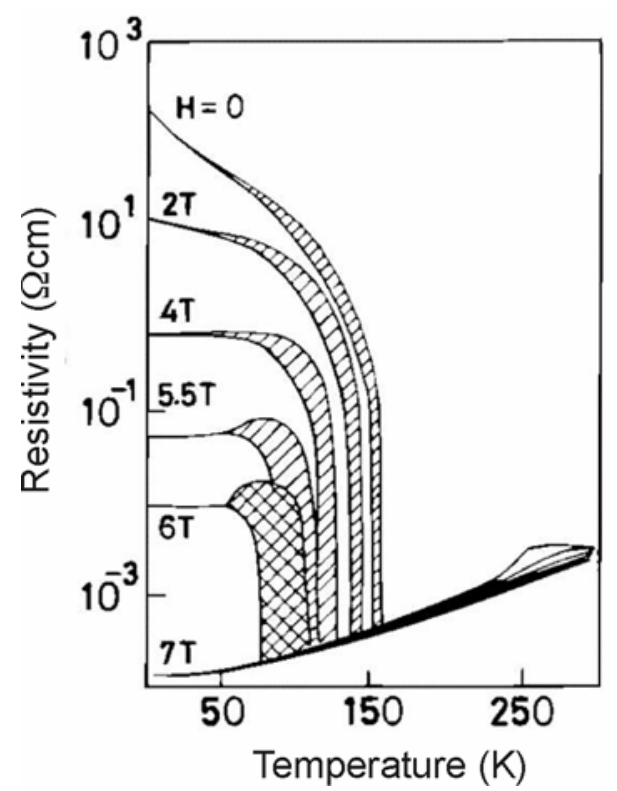

Figure 5. Effect of magnetic fields on the charge ordering transition of $\mathrm{Nd}_{0.5} \mathrm{Sr}_{0.5} \mathrm{MnO}_{3}$ (Kuwahara et al 1995).

AFM phase has a simultaneous ordering of charge, spins and orbitals in a complex spatial arrangement. The three phases coexist at low temperatures. The size scale of the inhomogeneities is atleast in the mesoscopic range (a few hundred nanometers or more), since they are large enough to produce well-defined reflections in neutron and X-ray diffraction patterns. The variation of the volume fraction of the three phases with temperature is shown in figure 9 (Woodward et al 1999). This phase diagram has an unusual feature with the three phases existing at the $150 \mathrm{~K}$ transition and below. The phase separation observed here is non-trivial in contrast to a situation where the sample is not phase-pure containing multiple phases that are both structurally and chemically distinct. Another interesting example of phase separation is in $\mathrm{Pr}_{0.7} \mathrm{Ca}_{0.3} \mathrm{MnO}_{3}$, which shows the presence of two distinct phases below the charge-ordering transition at $80 \mathrm{~K}$. A charge-ordered AFM phase and a charge-delocalized phase have been observed by neutron diffraction.

Signatures of electronic inhomogeneities may be inferred from magnetic and electron transport measurements as well. Thus, in $\left(\mathrm{La}_{1-x} \mathrm{Ln}_{x}\right)_{0.7} \mathrm{Ca}_{0.3} \mathrm{MnO}_{3}$, where $\mathrm{Ln}=\mathrm{Nd}$, Gd or Y, the lanthanide element was varied to control the average $A$-site cation radius $\left\langle r_{A}\right\rangle$ (Sudheendra and Rao 2003). In the case of the La compound $(x=0.0)$ with the largest value of $\left\langle r_{A}\right\rangle$, a clear FM transition with a saturation magnetization of $3 \mu_{\mathrm{B}}$ is observed up to a critical Nd doping of $x \leq x_{\mathrm{c}} \approx 0.5$ (figure 10a) (Sudheendra and Rao 2003). At higher values of $\mathrm{Nd}$ doping $\left(x>x_{\mathrm{c}}\right)$, there is a monotonic increase of the magnetization with decrease in temperature, although the magnetization does not attain the maximum value of $3 \mu_{\mathrm{B}}$. Reducing the value of $\left\langle r_{A}\right\rangle$ by Gd or Y (smallest value of $\left.\left\langle r_{A}\right\rangle\right)$ substitution, decreases the $x_{\mathrm{c}}$, i.e. the critical doping value $x$ above which the sharp FM transition vanishes, reduces with the mean $A$ site cation radius $\left\langle r_{A}\right\rangle$. Moreover, the FM transition temperature, $T_{\mathrm{c}}$, for $x<x_{\mathrm{c}}$ increases with $\left\langle r_{A}\right\rangle$ as expected. The reduction of the saturation magnetization at $x \geq x_{\mathrm{c}}$ is a signature of electronic and magnetic inhomogeneities induced by the $A$-site cation disorder. Transport measurements also show the signature of electronic inhomogeneities (figure 10b) (Sudheendra and Rao 2003).

All the $\left(\mathrm{La}_{1-x} \mathrm{Ln}_{x}\right)_{0.7} \mathrm{Ca}_{0.3} \mathrm{MnO}_{3}$ compositions with $\mathrm{Ln}=$ $\mathrm{Nd}, \mathrm{Gd}$ and $\mathrm{Y}$ exhibit a metal-insulator transition when $x<x_{\mathrm{c}}$. The insulator-metal transition temperature de- 
creases linearly on doping and increases nearly linearly with $\left\langle r_{A}\right\rangle$. Electronic phase separation in such manganates generally occurs when $\left\langle r_{A}\right\rangle$ is relatively small $(<1.18 \AA)$ and is favoured by size disorder arising from the size mismatch between the $A$-site cations. This disorder is quantified by the parameter $\sigma^{2}=\sum_{i} x_{i} r_{i}^{2}-\left\langle r_{A}\right\rangle^{2}$, where $x_{i}$ is the fractional $A$-site occupancy of species $i$ with radius $r_{i}$ (Rodriguez-Martinez and Attfield 2001). A study of several series of manganates with fixed $\left\langle r_{A}\right\rangle$ and varying $\sigma^{2}$ shows that, as $\sigma^{2}$ is lowered, the phase-separated system transforms to a FM metallic state. It has been demonstrated recently that in a series of manganates of the type, $\mathrm{Ln}_{0.7-x} \mathrm{Ln}_{x}^{\prime} \mathrm{A}_{0.3-y} \mathrm{~A}_{y}^{\prime} \mathrm{MnO}_{3}$, where $\left\langle r_{A}\right\rangle$ always remains large (thereby avoiding effects due to bandwidth), a decrease
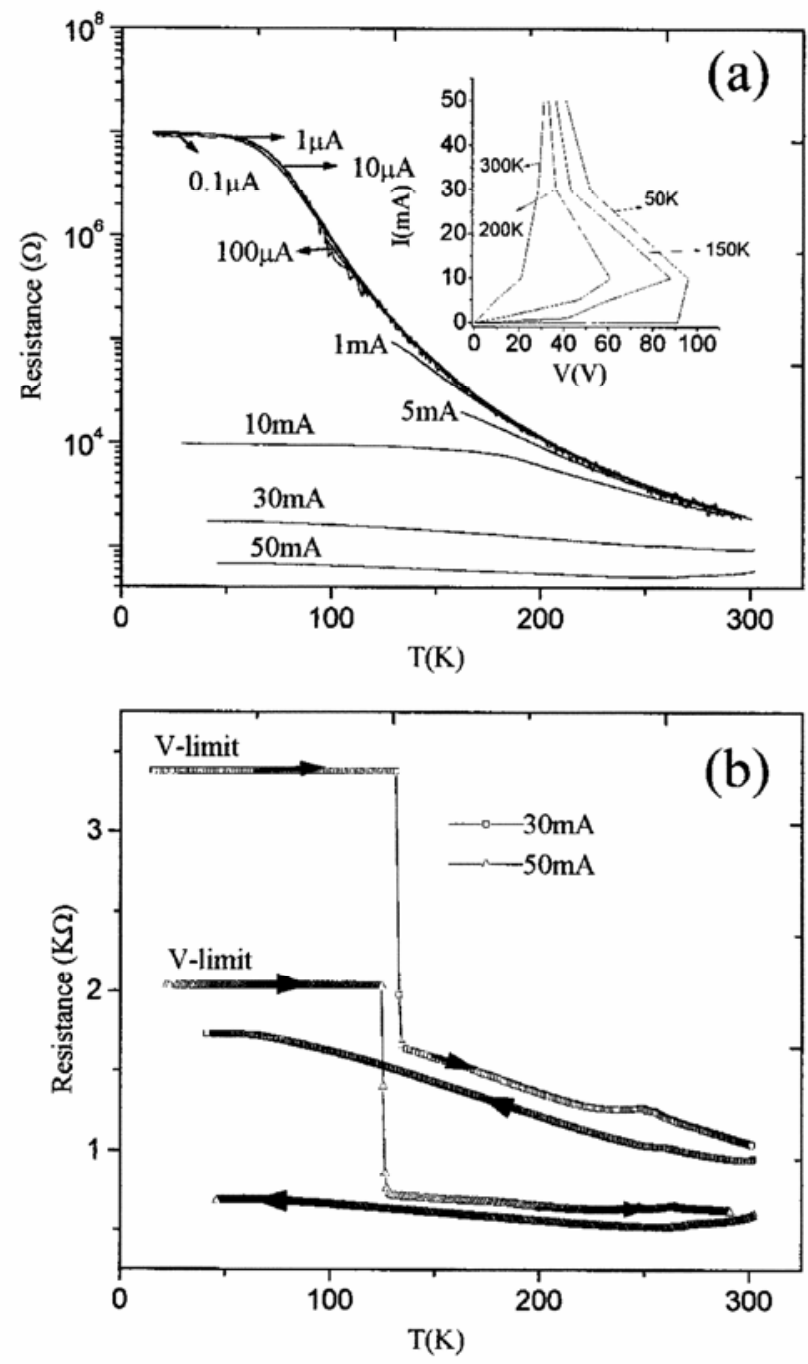

Figure 6. (a) Temperature variation of resistance of an oriented $\mathrm{Y}_{0.5} \mathrm{Ca}_{0.5} \mathrm{MnO}_{3}$ film deposited on $\mathrm{LaAlO}_{3}$ (100) for different values of the current and (b) resistance-temperature plots for two current values recorded over cooling and heating cycles showing memory effect. Inset in part a shows $I-V$ curves at different temperatures (Rao et al 2000). in $\sigma^{2}$ transforms the insulating non-magnetic state to a FM metallic state (Kundu et al 2005). The most important evidence for electronic phase separation in manganates comes from direct observational evidence obtained from images employing transmission electron microscopy, scanning probe microscopy and photoemission microspectroscopy (Shenoy and Rao 2008). Such images clearly provide a convincing case for the presence of the electronic inhomogeneities.

From the above discussion, it becomes clear that chargeordered manganates generally show electronic phase separation. The origin of electronic phase separation itself must be somewhat chemical in nature. This view is substantiated by the effect of size mismatch of the $A$-site cations or phase separation. The coexistence of different phases requires that the strain energies of the domains at the boundaries should be comparable. Since the different domains would have different electronic properties they can give rise to large dielectric constants (Sudheendra and Rao 2003). Thus, the high dielectric constant of $\mathrm{CaCu}_{3} \mathrm{Ti}_{4} \mathrm{O}_{12}$ is because of twin boundaries which may create a barrier layer capacitance (Subramanian et al 2000).

\subsection{Multiferroic nature of charge-ordered manganates}

It has been pointed out recently by Khomskii and coworkers (Efremov et al 2004) that coupling between magnetic and charge-ordering in charge-ordered and orbital-ordered perovskites can give rise to magnetoferroelectricity. As mentioned earlier, site-centred charge order (SCO) occurs around $x=0.5$ with a CE-type antiferromagnetic state while the bond-centred charge order (BCO) occurs around $x=0.4$ with a possible perpendicular spin structure. The composition with $x \sim 0.45$ is predicted to have a ferroelectric ground state.

We have investigated the dielectric properties, magnetocapacitance and related features of rare earth manganates of the general formula, $\mathrm{Ln}_{0.5} \mathrm{Ca}_{0.5} \mathrm{MnO}_{3}$ with $\mathrm{Ln}=\mathrm{Nd}$, Gd or $\mathrm{Y}$, exhibiting short range charge-ordering ( $\mathrm{SCO}$ ). We have also examined the manganate compositions of the type, $\mathrm{Ln}_{0.6} \mathrm{Ca}_{0.4} \mathrm{MnO}_{3}$, with $\mathrm{Ln}=\operatorname{Pr}$ and $\mathrm{Y}$, exhibiting BCO, which show canted antiferromagnetic or weak ferromagnetic behaviour at low temperatures due to electron phase separation (see figure $2 \mathrm{~b}$ ). They also show a broad maximum in the dielectric constant in the chargeordering region and exhibit magnetocapacitance on the application of a magnetic field of 1 or $2 \mathrm{~T}$. We shall now describe the results obtained with the $\mathrm{Y}_{1-x} \mathrm{Ca}_{x} \mathrm{MnO}_{3}$ and $\operatorname{Pr}_{0.6} \mathrm{Ca}_{0.4} \mathrm{MnO}_{3}$ compositions in some detail, since these manganates show the most robust charge-ordering.

\section{Results and discussion}

Nanoparticles of $\mathrm{Y}_{0.5} \mathrm{Ca}_{0.5} \mathrm{MnO}_{3}$ and $\operatorname{Pr}_{0.6} \mathrm{Ca}_{0.4} \mathrm{MnO}_{3}$ were prepared by the polymeric sol-gel method. The 


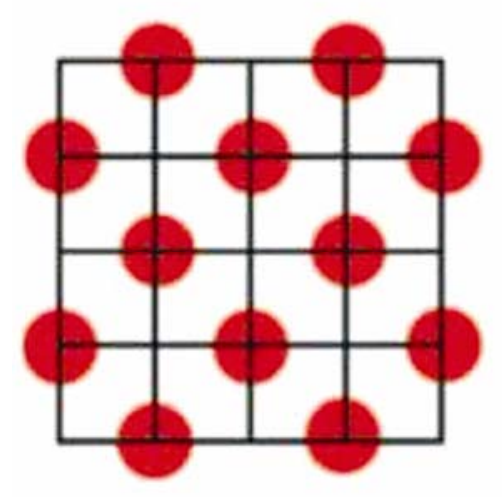

(a)

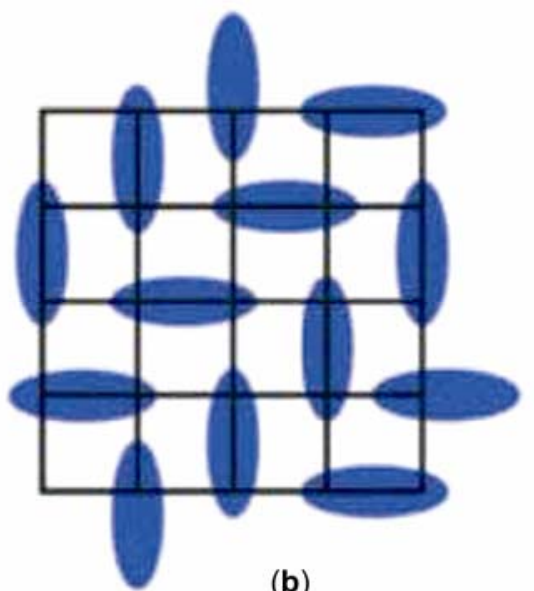

(b)

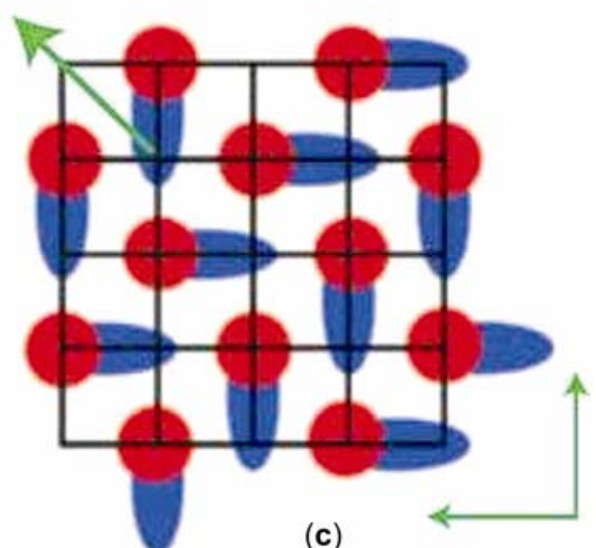

(c)

Figure 7. Three types of charge ordering: (a) site-centred charge order; (b) bond-centred charge order (the Zener polaron state); and (c) a ferroelectric intermediate state. The charge-ordered structure in $c$ lacks inversion symmetry. Thin green arrows indicate the dipole moments of horizontal and vertical dimers, and the diagonal arrow is the total ferroelectric moment (Efremov et al 2004).
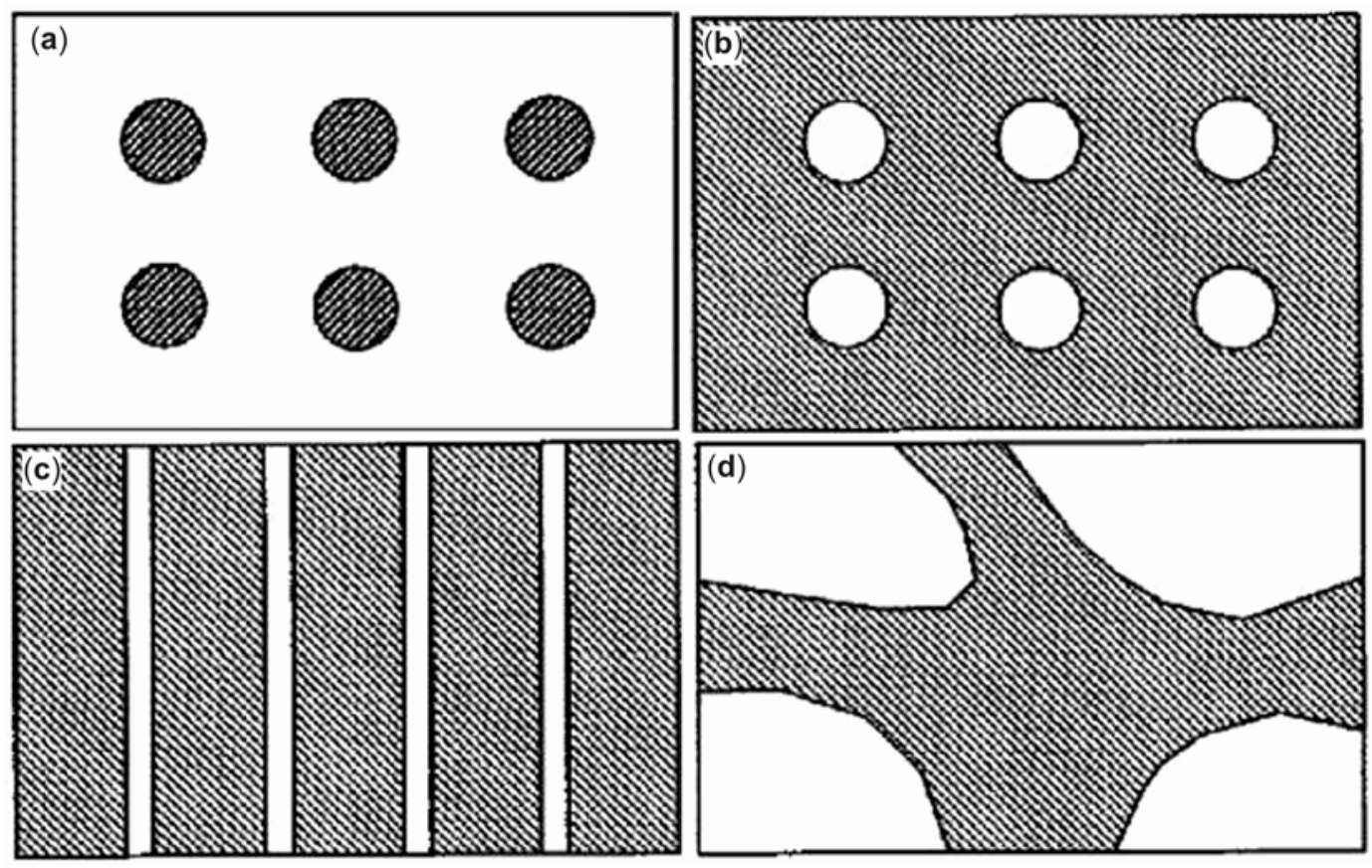

Figure 8. Schematic of electronic phase separation. Shaded portions indicate FM metallic regions; the unshaded portions correspond to AFM insulating regions. (a) FM metallic puddles in an insulating AFM background, (b) metallic regions with insulating droplets, (c) charged stripes and (d) phase separation on the mesoscopic scale.

nanoparticles with an average diameter of 30,70 and $110 \mathrm{~nm}$ were prepared by heating at 600,800 and $1000^{\circ} \mathrm{C}$ as evidenced from FESEM and TEM. The XRD patterns show greater broadening of the peaks in the samples prepared at lower temperatures.

The nanoparticles exhibit magnetic properties drastically different from the bulk samples prepared at high temperatures. The small particles do not show the chargeordering transition or the antiferromagnetic transition.
Instead, they show evidence for prominent ferromagnetic interaction, a feature that has been found in small particles of other charge-ordered rare earth manganates as well (Sudheendra et al 2002; Rao et al 2006; Zhang et al 2007). Our studies show that upon decreasing the particle sizes the ferromagnetic phase dominates over the antiferromagnetic phases. $\operatorname{Pr}_{0.6} \mathrm{Ca}_{0.4} \mathrm{MnO}_{3}$ samples are much more ferromagnetic than the $\mathrm{Y}_{0.5} \mathrm{Ca}_{0.5} \mathrm{MnO}_{3}$ samples because of the presence of the extra electron on the $\mathrm{Mn}^{+4}$ 
sites which increase the ferromagnetic interaction in these samples.

$\mathrm{Y}_{0.5} \mathrm{Ca}_{0.5} \mathrm{MnO}_{3}$ exhibits a charge-ordering transition at $275 \mathrm{~K}\left(T_{\mathrm{CO}}\right)$ and an antiferromagnetic transition at $110 \mathrm{~K}$ $\left(T_{\mathrm{N}}\right)$ (Arulraj et al 1998). Ferromagnetic interactions are manifested at low temperatures due to electronic phase

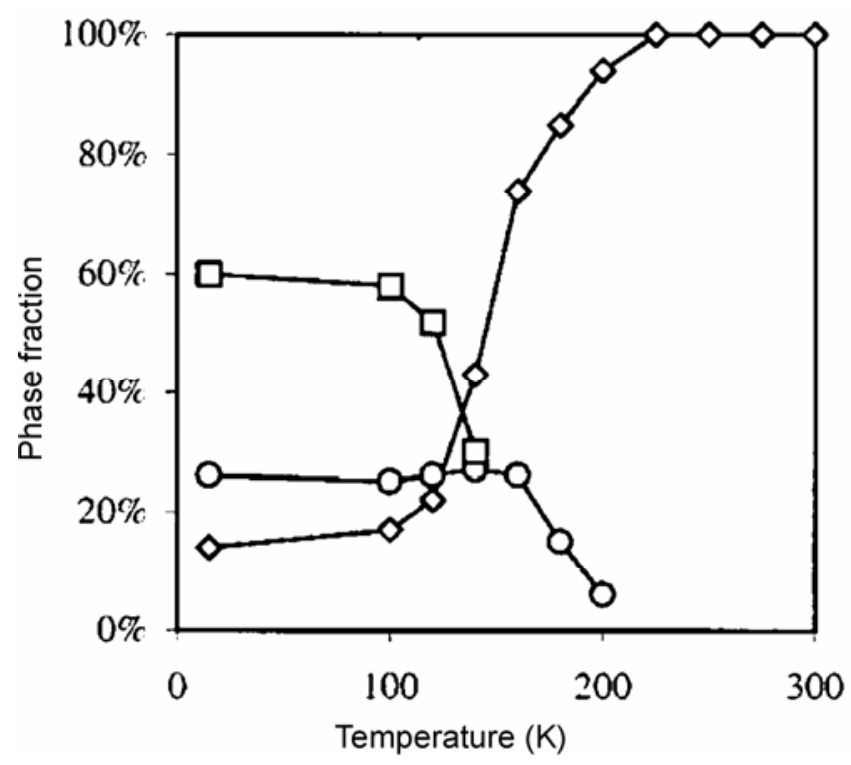

Figure 9. Variation in the percentage of the different phases of $\mathrm{Nd}_{0.5} \mathrm{Sr}_{0.5} \mathrm{MnO}_{3}$ with temperature: FMM phase (diamonds); orbitally ordered $A$-type AFM phase (circles); charge-ordered CE-type AFM phase (squares) (Woodward et al 1999).
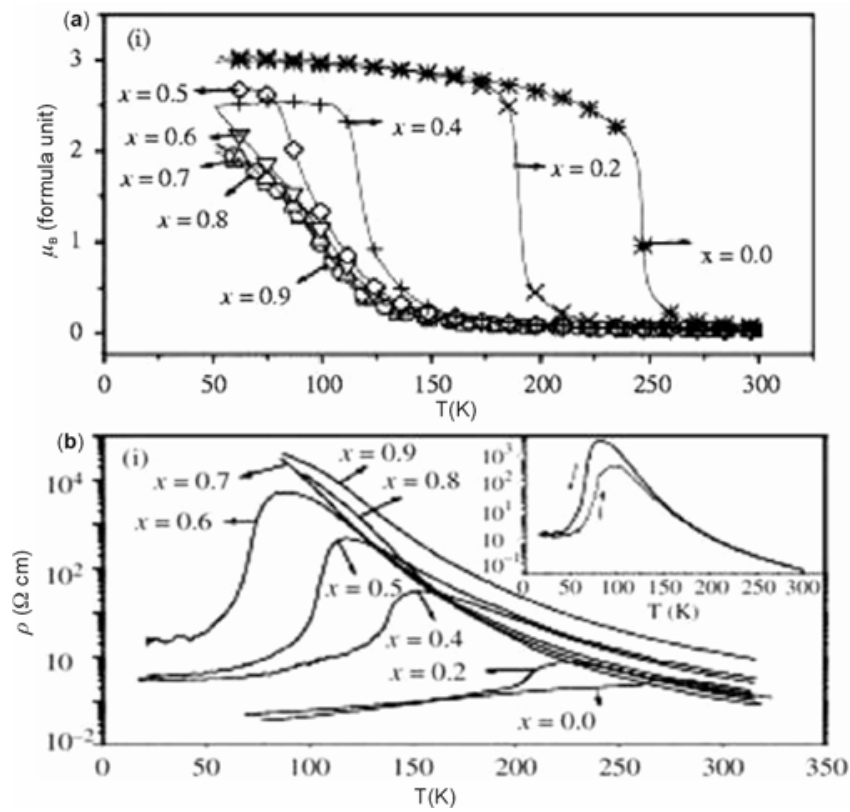

Figure 10. Temperature dependence of (a) the effective magnetic moment per formula unit and (b) the resistivity in $\left(\mathrm{La}_{1-x}\right.$ $\left.\mathrm{Nd}_{x}\right)_{0.7} \mathrm{Ca}_{0.3} \mathrm{MnO}_{3}$. The insets show the 'resistivity hysteresis' upon warming of the sample (Sudheendra and Rao 2003). separation (Shenoy et al 2006; Shenoy and Rao 2008). Magnetic hysteresis is observed at $T<20 \mathrm{~K}$, the values of remnant magnetization $\left(M_{\mathrm{r}}\right)$ and coercive field $\left(H_{\mathrm{C}}\right)$ at $5 \mathrm{~K}$ being $35 \mathrm{emu} / \mathrm{mol}$ and $489 \mathrm{Oe}$, respectively. In figure

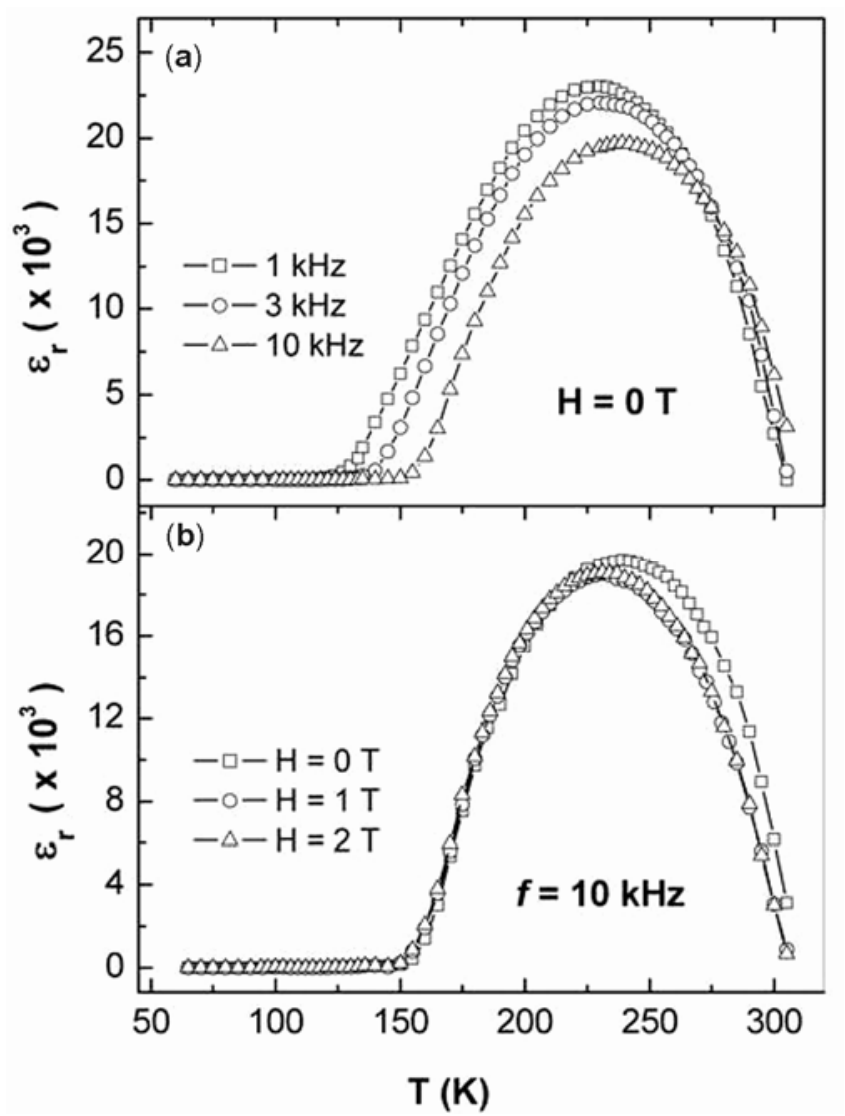

Figure 11. (a) Temperature-dependence of the dielectric constant of $\mathrm{Y}_{0.5} \mathrm{Ca}_{0.5} \mathrm{MnO}_{3}$ at different frequencies and (b) effect of magnetic fields on the dielectric behaviour of $\mathrm{Y}_{0.5} \mathrm{Ca}_{0.5} \mathrm{MnO}_{3}$ at $10 \mathrm{kHz}$.

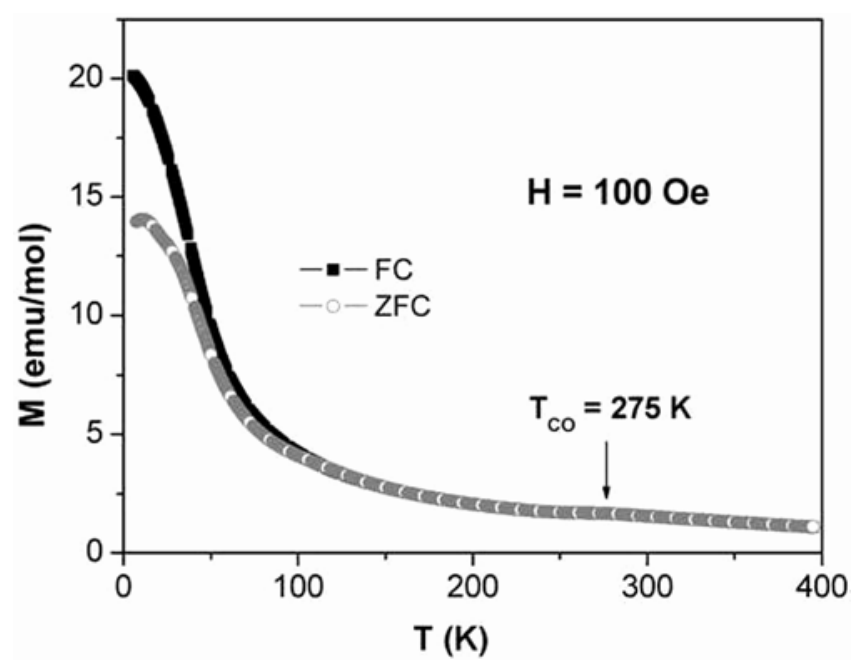

Figure 12. Variation of field-cooled (FC) and zero-fieldcooled (ZFC) magnetizations of $\mathrm{Y}_{0.55} \mathrm{Ca}_{0.45} \mathrm{MnO}_{3}$ as a function of temperature at a magnetic field of 100 Oe showing the charge-ordering transition at $275 \mathrm{~K}$. 
11(a), we have plotted the dielectric constant as a function of temperature for various frequencies. The dielectric constant exhibits a maximum in the $225-240 \mathrm{~K}$ range. On

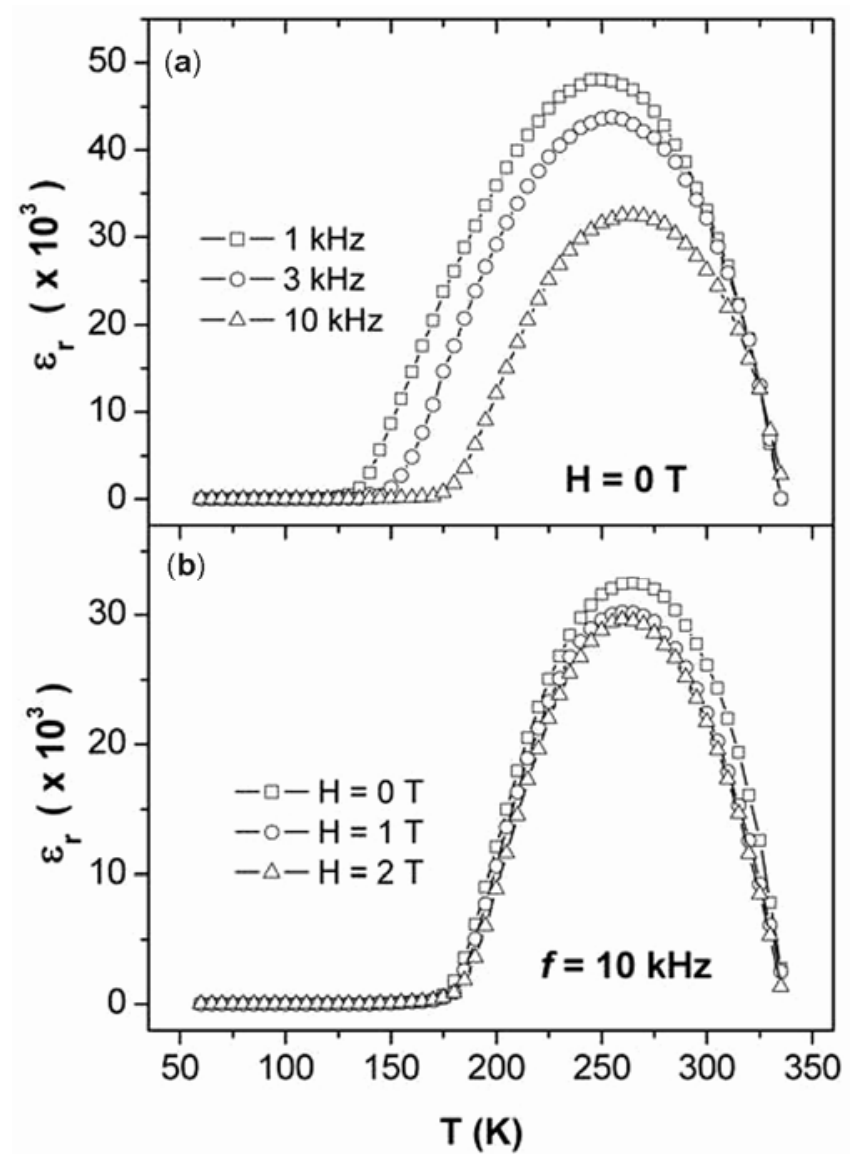

Figure 13. (a) Temperature-dependence of the dielectric constant of $\mathrm{Y}_{0.55} \mathrm{Ca}_{0.45} \mathrm{MnO}_{3}$ at different frequencies and (b) effect of magnetic fields on the dielectric behaviour of $\mathrm{Y}_{0.55} \mathrm{Ca}_{0.45}$ $\mathrm{MnO}_{3}$ at $10 \mathrm{kHz}$.

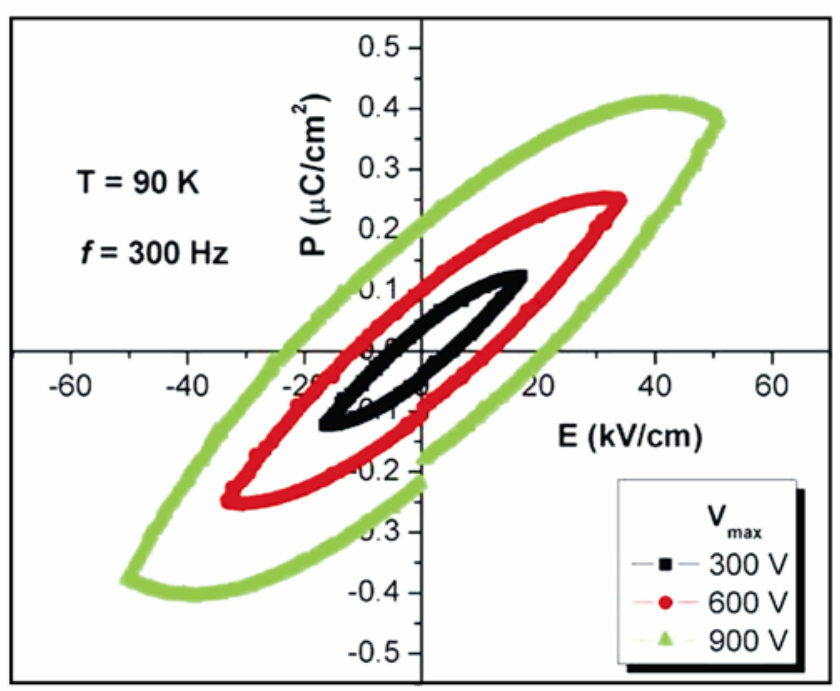

Figure 14. Ferroelectric hysteresis loops of a thin pellet of $\mathrm{Y}_{0.55} \mathrm{Ca}_{0.45} \mathrm{MnO}_{3}(300 \mathrm{~Hz})$ at $90 \mathrm{~K}$ for various voltages. increasing the frequency, the dielectric constant decreases markedly with the maximum shifting to slightly higher temperatures. At $10 \mathrm{kHz}$, the maximum value of the dielectric constant is $2 \times 10^{4}$, which is high compared to other rare-earth manganates. This would be expected considering the robust charge-ordered state and small size of the yttrium ion. The ferroelectric transition temperature $\left(T_{\mathrm{CE}}\right)$ derived from the inverse Curie-Weiss plot of dielectric constant is close to $T_{\mathrm{CO}}$. Application of a magnetic field of $1 T$ has a significant effect on the dielectric constant causing a shift of the dielectric constant maximum to a lower temperature as can be seen in figure 11(b).

Magnetic measurements on $\mathrm{Y}_{0.55} \mathrm{Ca}_{0.45} \mathrm{MnO}_{3}$ show evidence for charge ordering at $275 \mathrm{~K}$ and weak ferromagnetic interactions below around $50 \mathrm{~K}$ (figure 12). It exhibits magnetic hysteresis loop at low temperatures, the values of $M_{\mathrm{r}}$ and $H_{\mathrm{C}}$ at $5 \mathrm{~K}$ being $45 \mathrm{emu} / \mathrm{mol}$ and $502 \mathrm{Oe}$, respectively. We show the temperature variation
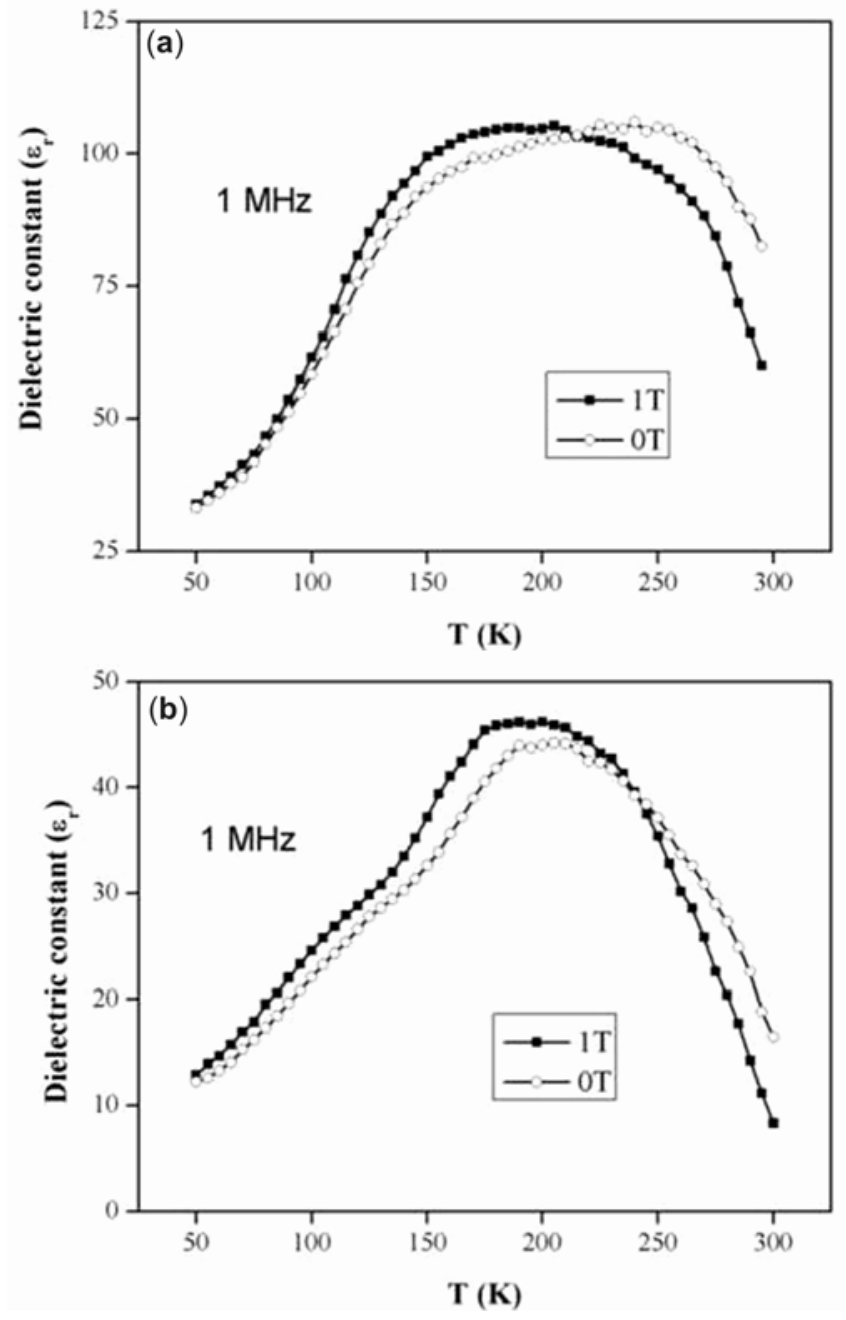

Figure 15. Effect of magnetic field on the temperature dependence of dielectric constant on nanoparticles of $\mathrm{Y}_{0.5} \mathrm{Ca}_{0.5}$ $\mathrm{MnO}_{3}$ prepared at $(\mathbf{a}) 600^{\circ} \mathrm{C}$ and $(\mathbf{b}) 800^{\circ} \mathrm{C}$. 
of the dielectric constant for $\mathrm{Y}_{0.55} \mathrm{Ca}_{0.45} \mathrm{MnO}_{3}$ in figure 13(a). Based on the Curie-Weiss plot, we estimate $T_{\mathrm{CE}}$ to be $326 \mathrm{~K}$ which is much higher than that of $\mathrm{Y}_{0.5} \mathrm{Ca}_{0.5} \mathrm{MnO}_{3}$. The maximum value of dielectric constant observed at $10 \mathrm{kHz}$ is $3.3 \times 10^{4}$. The effect of magnetic field on the dielectric constants is shown in figure 13(b). The data show clear evidence for magnetocapacitance. $\mathrm{Y}_{0.6} \mathrm{Ca}_{0.4}$ $\mathrm{MnO}_{3}$, with bond-centred charge ordering (BCO), also shows the properties similar to $\mathrm{Y}_{0.5} \mathrm{Ca}_{0.5} \mathrm{MnO}_{3}$ with a $T_{\mathrm{CE}}$ of $300 \mathrm{~K}$ and magnetocapacitance at $1 \mathrm{~T}$.

Since most of the rare-earth manganates possess relatively high conductivity, the dielectric hysteresis loops exhibited by them are similar to those of leaky dielectrics (Scott 2008). The dielectric hysteresis loop of a very thin sample (thickness, $\sim 180 \mu \mathrm{m}$ ) of $\mathrm{Y}_{0.55} \mathrm{Ca}_{0.45} \mathrm{MnO}_{3}$ (see figure 14) recorded by us shows a maximum polarization of around $0.43 \mu \mathrm{C} / \mathrm{cm}^{2}$ and a remnant polarization of $0.25 \mu \mathrm{C} / \mathrm{cm}^{2}$ at $900 \mathrm{~V}$. In view of the possible unreliability of dielectric hysteresis loops (Scott 2008), we have
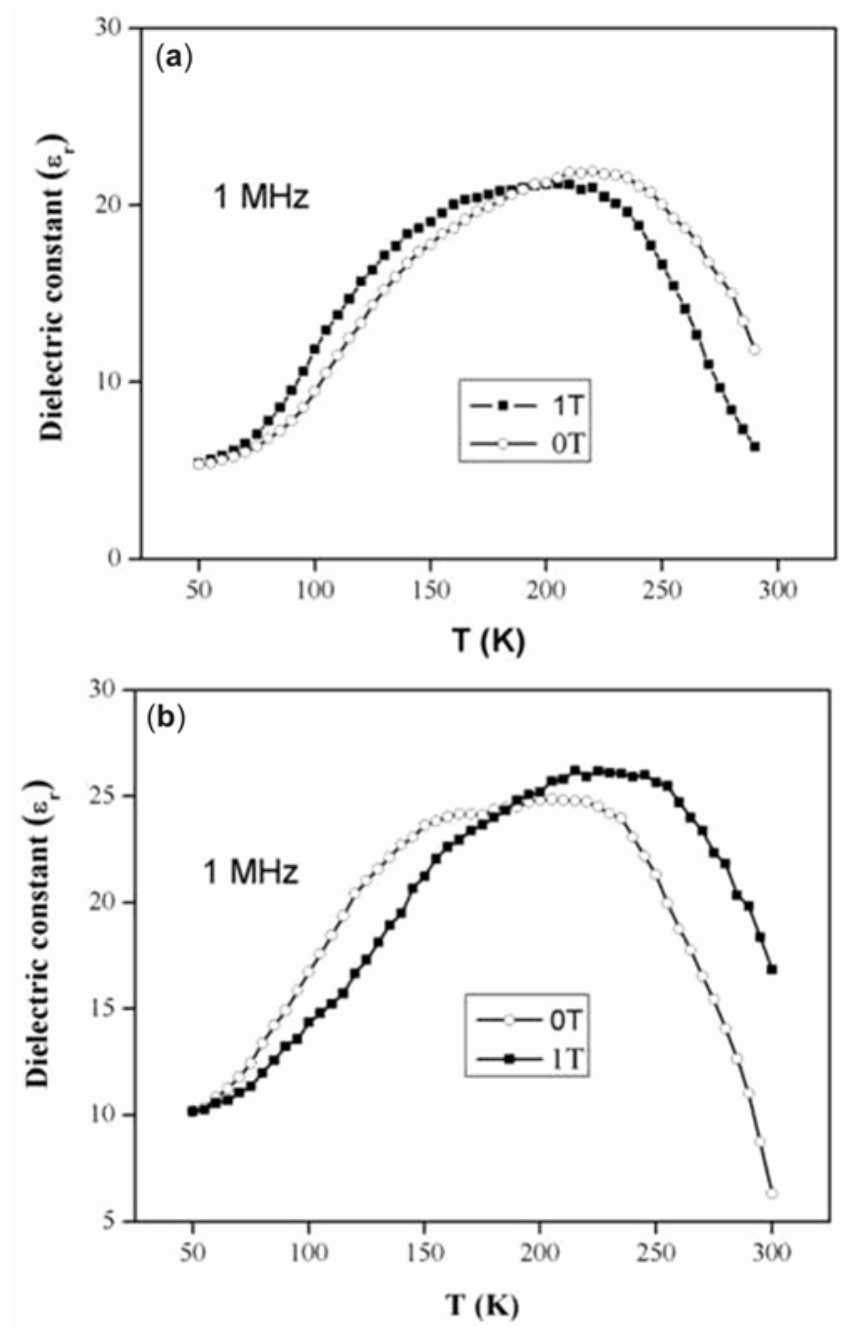

Figure 16. Effect of magnetic field on the temperature dependence of dielectric constant on nanoparticles of $\operatorname{Pr}_{0.6} \mathrm{Ca}_{0.4} \mathrm{MnO}_{3}$ prepared at (a) $600^{\circ} \mathrm{C}$ and (b) $800^{\circ} \mathrm{C}$. examined second harmonic generation (SHG) by polycrystalline samples of $\mathrm{Y}_{1-x} \mathrm{Ca}_{x} \mathrm{MnO}_{3}$ with $x=0.45$ and $0 \cdot 4$. The SHG responses of $\mathrm{Y}_{0.55} \mathrm{Ca}_{0.45} \mathrm{MnO}_{3}$ and $\mathrm{Y}_{0.6} \mathrm{Ca}_{0.4}$ $\mathrm{MnO}_{3}$ were found to be 1.25 and 0.79 times that of quartz, respectively. These results establish that these chargeordered manganates do indeed possess non-linear properties and polarization features of ferroelectrics.

In figures 15(a) and (b), we show the temperature variation of dielectric constant at $1 \mathrm{MHz}$ with and without the application of the magnetic field (1T) for nanoparticles of $\mathrm{Y}_{0.5} \mathrm{Ca}_{0.5} \mathrm{MnO}_{3}$ heated at 600 and $800^{\circ} \mathrm{C}$, respectively. The dielectric constant of the $\mathrm{Y}_{0.5} \mathrm{Ca}_{0.5} \mathrm{MnO}_{3}$ samples are much lower (by order of 1000) than the bulk samples. The magnetic field has a significant effect on the dielectric constant, the effect occurring over a wide temperature range of $75-300 \mathrm{~K}$. Both the nano samples show positive magnetocapacitance below charge ordering transition temperature and negative after that as observed in the bulk samples.

In figures 16(a) and (b), we show the magnetocapacitive effect in $\mathrm{Pr}_{0.6} \mathrm{Ca}_{0.4} \mathrm{MnO}_{3}$ at $1 \mathrm{MHz}$. The value of the dielectric constant is 3 orders less than the bulk samples (Serrao et al 2007; Sahu et al 2009). These samples also show a transition in broad temperature range. The nano samples prepared at $600^{\circ} \mathrm{C}$ show positive magnetocapacitance while those prepared at $800^{\circ} \mathrm{C}$ negative magnetocapacitance below $188 \mathrm{~K}$ and opposite after that. While the trend observed in the case of 600 sample is opposite to that of the bulk, the 800 samples show the same trend as the bulk.

\section{Conclusions}

Magnetocapacitance exhibited by these rare earth manganates is genuine since we do not observe any change in

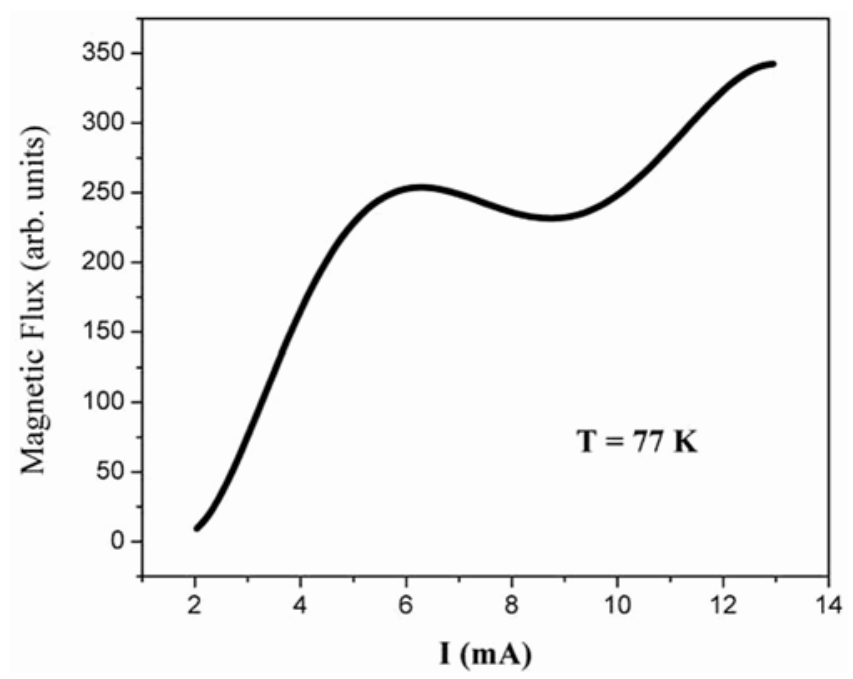

Figure 17. Magnetic flux (SQUID signal) at $77 \mathrm{~K}$ as a function of biasing current through the sample (Sudheendra et al 2002). 
the resistance on the application of magnetic fields up to $7 \mathrm{~T}$ in the case of $\mathrm{Y}_{1-x} \mathrm{Ca}_{x} \mathrm{MnO}_{3}$. Furthermore, these materials show a magnetic flux on the application of electric fields (Guha et al 2000; Sakai and Tokura 2008) (figure 17), indicating that there is interaction between the electric and magnetic order parameters. This would be expected since the metallic state created by the electric field should necessarily be ferromagnetic according to the DE mechanism. All these observations establish that the charge-ordered rare earth manganates, specifically the $\mathrm{Y}_{1-x} \mathrm{Ca}_{x} \mathrm{MnO}_{3}$ compositions, are not only multiferroic but also magnetoelectric. These observations are consistent with the predictions of Efremov et al (2004). The magnetocapacitive behaviour of rare earth manganates may reflect the electronic phase-separation present in these materials wherein charge-ordered and ferromagnetic regimes or domains of different sizes coexist.

\section{Acknowledgements}

The authors thank Professor C N R Rao for suggesting the problem and guidance. One of us (JRS) thanks the University Grants Commission, Government of India, for a fellowship.

\section{References}

Arulraj A, Gundakaram R, Biswas A, Gayathri N, Raychaudhuri A K and Rao C N R 1998 J. Phys.: Condens. Matter 10 4447

Cheong S W and Mostovoy M 2007 Nat. Mater. 613

Dagotto E 2005 Science 309257

Eerenstein W, Mathur N D and Scott J F 2006 Nature 442 759

Efremov D V, van den Brink J and Khomskii D I 2004 Nat. Mater. 3853

Guha A, Khare N, Raychaudhuri A K and Rao C N R 2000 Phys. Rev. B62 R11941

Kimura T, Goto T, Shintani H, Ishizaka K, Arima T and Tokura Y 2003 Nature 42655

Kundu A K, Seikh M M, Ramesha K and Rao C N R 2005 J. Phys.: Condens. Matter 174171

Kuwahara H, Tomioka Y, Asamitsu A, Moritomo Y and Tokura Y 1995 Science 270961
Lees M R, Barratt J, Balakrishnan G, Paul D McK and Yethiraj M 1995 Phys. Rev. B52 R14303

Prellier W, Singh M P and Murugavel P 2005 J. Phys.: Condens. Matter 17 R803

Ramesh R and Spaldin N A 2007 Nat. Mater. 621

Rao C N R 2000 J. Phys. Chem. B104 5877

Rao C N R and Raveau B (eds) 1998 Colossal magnetoresistance, charge ordering and related properties of manganese oxides (Singapore: World Scientific)

Rao C N R and Serrao C R 2007 J. Mater. Chem. 174931

Rao C N R, Raju A R, Ponnambalam V, Parashar S and Kumar N 2000 Phys. Rev. B61 594

Rao S S, Tripathi S, Pandey D and Bhat S V 2006 Phys. Rev. B74 14416

Rodriguez-Martinez L M and Attfield J P 2001 Phys. Rev. B63 024424

Sahu J R, Serrao C R, Ghosh A, Sundaresan A and Rao C N R 2009 Solid State Commun. 14949

Sakai H and Tokura Y 2008 Appl. Phys. Lett. 92102514

Salamon M B and Jaime M 2001 Rev. Mod. Phys. 73583

Scott J F 2008 J. Phys.: Condens. Matter 20021001

Serrao C R, Sundaresan A and Rao C N R 2007 J. Phys.: Condens. Matter 19496217

Seshadri R and Hill N A 2001 Chem. Mater. 132892

Shenoy V B and Rao C N R 2008 Philos. Trans. R. Soc. $\mathbf{A 3 6 6}$ 63

Shenoy V B, Sarma D D and Rao C N R 2006 Chem. Phys. Chem. 72053

Subramanian M A, Li D, Duan N, Reisner B A and Sleight A W 2000 J. Solid State Chem. 151323

Sudheendra L and Rao C N R 2003 J. Phys.: Condens. Matter 153029

Sudheendra L, Chinh H D, Raju A R, Raychaudhuri A K and Rao C N R 2002 Solid State Commun. 12253

Tokura Y 2006 Rep. Prog. Phys. 69797

Tomioka Y, Asamitsu A, Kuwahara H, Moritomo Y and Tokura Y 1996 Phys. Rev. B53 1689

Van Aken B B, Palstra T T M, Filippetti A and Spaldin N A 2004 Nat. Mater. 3164

Vogt T, Cheetham A K, Mahendiran R, Raychaudhuri A K, Mahesh R and Rao C N R 1996 Phys. Rev. B54 15303

Wollan E O and Koehler W C 1955 Phys. Rev. 100545

Woodward P M, Vogt T, Cox D E, Arulraj A, Rao C N R, Karen P and Cheetham A K 1998 Chem. Mater. 103652

Woodward P M, Cox D E, Vogt T, Rao C N R and Cheetham A K 1999 Chem. Mater. 113528

Zhang T, Zhou T F, Qian T and Li X G 2007 Phys. Rev. B76 174415 